\title{
InPEX OF ADVERUISERS
}

4pi Analysis, Inc (www.4pi.com)

Advanced Microscopy Techniques(www.amtimaging.com)

Australian Microscopy Meeting.

CamScan USA (www.camscan-Lsa.com)

EBSciences (ebs@ebsciences.com).

EDAX Inc.(www.edax.com)

EMPIX Imaging (www.empix.com)

Ernest F. Fullam (www.fullam.com)

FEI Company (www.feic.com)

Gatan Inc. (www.gatan.com)

Geller MicroAnalytical Lab (www.gellermicro.com)

Hitachi High Technologies America (www.hitachi-hhta.com)

Imago Scientific Instruments Corporation, (www. imago.com)

IXRF Systems (Www.ixfsystems.com)

JEOL USA (www.jeol.com)

LEO Electron Microscopy (www.leo-usa.com).

M.E. Taylor Engineering (www.semsupplies.com)

Materials Analytical Services (MAS) (www. mastest.com)
...37 McCrone Research Institute (mcri.org) 31

41 Meiji Techno America (www.meijitechno.com) .......................................30

.57 Micro Star Technologies (www. Microstartech.com) ...............................25

15 Minitool Micro Precision Products (www.minitoolinc.com)........................53

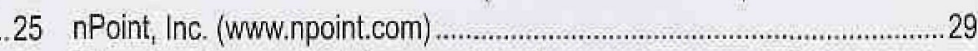

19 Princeton Gamma Tech (www.pgt.com) …......................................4

59 Reindeer Graphics, Inc (reindeergraphics.com)...........................12, 49

21 RMC Products (www. rmcproducts. com) ….......................................59

.2 ScopeTronix Quality Products (Www.scopetronix.com) ............................49

.13 SensIR Technologies (www. sensir.com/objective) ...........................22, 23

.47 Small World (www.small-world.net) ..................................................47

.63 Soft Imaging Systems Corporation (www.soft-imaging.com) _..................21

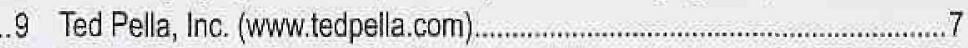

39. Thermo Electron Corporation (www. thermo.com/nss) ............................33

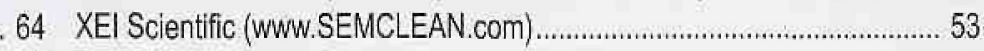

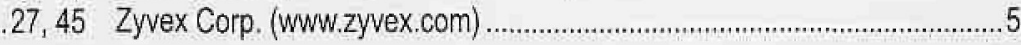

59 Employment Advertisements

36 Saint Lawrence University...

(1)

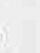

.

(1)

